\title{
Spectral diagnostics with the SDO EVE flare lines ${ }^{\star}$
}

\author{
G. Del Zanna ${ }^{1}$ and T. N. Woods ${ }^{2}$ \\ 1 DAMTP, Centre for Mathematical Sciences, Wilberforce road, Cambridge CB3 OWA, UK \\ e-mail: g.del-zanna@damtp.cam.ac.uk \\ ${ }^{2}$ Laboratory for Atmospheric and Space Physics, University of Colorado, Boulder, Colorado 20305, USA
}

Received 21 December 2012 / Accepted 5 April 2013

ABSTRACT

\begin{abstract}
The diagnostic use of the soft X-ray and EUV lines observed with the Solar Dynamics Observatory (SDO) Extreme ultraviolet Variability Experiment (EVE) is discussed. We focus on all the flare lines observed in the 80-640 ̊ range (mainly due to Fe XVIII-Fe XXIV), showing their use to measure temperatures, emission measures, densities, and chemical abundances. We discuss their identification at the EVE resolution, by using the latest atomic data, and by assessing possible sources of blending, taking into account higher-resolution solar spectra. We present observations of four flares, and study in more detail the gradual phase peak of the 7 March 2012 X5.6 flare. Good agreement between observations and theory is found in most cases, and the best lines for diagnostics are recommended. We found reasonable densities $\left(10^{11.2} \mathrm{~cm}^{-3}\right.$ from Fe XXI lines), but isothermal temperatures (12 MK) lower than those estimated with GOES. We show that EVE can be used to measure relative elemental abundance, and find photospheric argon/iron and calcium/iron abundances. We also show that lines normally formed in the quiet Sun in the low transition region such as $\mathrm{O}$ III are the best to study the impulsive phase.
\end{abstract}

Key words. line: identification - techniques: spectroscopic - Sun: flares - Sun: abundances - atomic data - Sun: corona

\section{Introduction}

The soft X-ray region of the spectrum (60-160 $\AA$ ) contains the brightest $n=2 \rightarrow n=2$ transitions from Fe XVIII-Fe XXIII (the flare lines). These transitions have been studied extensively in the laboratory (see, e.g. Kononov et al. 1976; Stratton et al. 1984), partly because they offer many line ratios that can be used to measure electron densities. Accurate wavelength measurements are also from laboratory spectra, for example from Sugar \& Rowan (1995) for lines from the Fe XX-Fe XXIII ions.

Kastner et al. (1974, hereafter K74) reported the first solar spectra (taken in 1969 from OSO-5) and some identifications in the 94-140 $\AA$ wavelength region, where strong flare resonance lines from a range of iron ions is present. These iron lines provide a range of diagnostic possibilities, especially of measuring temperatures and densities of the flare plasma. Fawcett \& Cowan (1975) later revised the Kastner et al. (1974) identifications, and added several new ones. A good overview of the OSO-5 lines, the relevant atomic data and the diagnostic possibilities of measuring electron densities is given by Mason et al. (1984).

The $n=2 \rightarrow n=2$ transitions from Fe XVIII-Fe XXIII have also been observed in spectra of active stars with the Extreme UltraViolet Explorer (EUVE) satellite, and have been used to provide electron densities and temperatures as described for example in Del Zanna (1995), later summarised in Monsignori Fossi et al. (1996). Since 1999, the soft X-ray spectral region has been observed with the Chandra Low Energy Transmission Grating (LETG). The flare lines are prominent in active stars (see, e.g. Mewe et al. 2001).

The Solar Dynamics Observatory (SDO) Atmospheric Imaging Assembly (AIA, see Lemen et al. 2012) has been

\footnotetext{
$\star$ Appendix $\mathrm{A}$ is available in electronic form at http: //www . aanda.org
}

producing stunning broad-band extreme-ultraviolet (EUV) images of the Sun since May 2010, while the Extreme ultraviolet Variability Experiment (EVE; Woods et al. 2012) has been producing spectra in the $60-1050 \AA$ range with a resolution of approximately $1 \AA$ and a nominal cadence of $10 \mathrm{~s}$. The MEGS-A (60-370 $\AA$ ) has been operating routinely, while the MEGS-B channel (370-1060 A) has suffered significant degradation above $700 \AA$ and is normally used for observing at lower cadence.

The EVE spectrometer has a potential for a range of diagnostics, only some of which have been explored so far. For the first time it is possible to study the evolution of flares with high cadence using the soft X-rays and EUV lines. For example, Chamberlin et al. (2012) studied the temperature evolutions of different types of flares. Petkaki et al. (2012) presented a detailed study of a small flare, producing electron temperatures from the soft X-ray lines for the first time. Estimates of electron densities have also been made by Milligan et al. (2012b). Milligan et al. (2012a) pointed out the use of the free-free and free-bound continua for diagnostics, while Hudson et al. (2011) pointed out that Doppler measurements are also possible.

The main aim of this paper is to assess the possible diagnostic use of the soft X-ray and EUV flare lines in the EVE spectra, mostly from Fe XVIII-Fe XXIV, to measure temperatures, emission measures (EM), densities, and chemical abundances. This is done by discussing their identification at the EVE resolution, using the latest atomic data, and by assessing possible sources of blending. The enhancements of the flare lines are large, but flare sizes are very small compared to the whole solar disk, so enhancements in the EVE full-disk spectra are very small, often of the order of 10-30\% over the background (pre-flare) solar spectrum. It has been common practice in EVE flare studies to subtract a pre-flare spectrum, with the assumption that the lines 
present during the pre-flare phase would not change during the flare. This is not a very good assumption if the lines of the preflare spectrum are not known, as discussed here.

One problem with the soft X-rays and EUV is that it is a very crowded spectral region, but we have not had solar spectra with sufficiently high resolution to study them in detail over the full $\mathrm{X}$-ray and EUV ranges. Significant advances in the EUV have been made recently thanks to the high-resolution Hinode EIS spectra (see, e.g. the atlas of coronal lines in Del Zanna 2012c).

Within the soft X-rays, Manson (1972, hereafter M72) provided an excellent list of calibrated soft X-ray irradiances observed in quiet and active (but not flaring) conditions in the 30-130 A range, at medium resolution (0.16-0.23 ̊). Excellent agreement between the quiet Sun spectrum and the EVE prototype spectrum was found below $100 \AA$ (Del Zanna 2012b). The M72 quiet Sun spectrum above $100 \AA$ was recalibrated and has been considered for the present analysis. Behring et al. (1972, hereafter Be72) published a list of lines in the $60-385 \AA$ region observed with high resolution $(0.06 \AA)$; however, only the strongest lines were listed, and the intensities were not calibrated. Malinovsky \& Heroux (1973, hereafter MH73) presented irradiances in the 50-300 $\AA$ range with a medium resolution $(0.25 \AA)$; these, however, turned out to be incorrectly calibrated, as explained in Del Zanna (2012b). The MH73 spectrum has been recalibrated and has been considered for the present analysis.

Another problem with the soft X-rays has been the lack of atomic data. The soft X-rays are dominated by millions of $n=4 \rightarrow n=3$ transitions from Fe VIII-Fe XVI but almost no atomic data were available, for the Fe VIII-Fe XIV ions. These calculations turned out to be far more complex than anticipated (as discussed in Del Zanna et al. 2012b), but have been done within the UK-funded APAP ${ }^{1}$ network (O'Dwyer et al. 2012; Del Zanna et al. 2012a,b; Del Zanna \& Storey 2012, 2013; Liang et al. 2010). A new calculation for Fe XVI has also been done (Liang et al. 2009). Some of the new atomic data have been made available via the CHIANTI v.7.1 release in Sept 2012 (Landi et al. 2013). We use these CHIANTI data for the present analysis, including the ion fractions, calculated under the assumption of ionization equilibrium.

A major problem in the soft X-rays has been the lack of line identifications, in particular for the iron lines. The identification of the iron soft X-rays $n=4 \rightarrow n=3$ transitions started with the pioneering work by Edlén in the 1930s (see, e.g. Edlén 1937 on Fe X). Edlén's work was extended to the iron $3 \mathrm{~s}^{2} 3 \mathrm{p}^{2} 4 l(l=\mathrm{s}, \mathrm{p}, \mathrm{d}, \mathrm{f})$ levels by the fundamental laboratory work of Fawcett et al. (1972). Fawcett's plates have been revisited, in light of the new atomic calculations, and several new identifications (including the strongest lines in the soft X-rays) have been presented in Del Zanna (2012b), where comparisons with solar spectra were also given. The new identifications of the main lines have been made available via the CHIANTI v.7.1 release (Landi et al. 2013). Despite these advances, a significant fraction of the soft X-ray lines are still unidentified.

\section{Observations and results}

We have analysed several flares of different classes. Our preliminary analysis used the publicly-available version 2 of the EVE level 2 data products. Several discrepancies between predicted and observed line intensities were found in the 2012 observations. The same analysis has been later performed on the

\footnotetext{
http://www . apap-network . org
}

version 3 data, which overall show much better agreement with theory. The changes between version 2 and 3 are significant, with the irradiances in several lines around $100 \AA$ decreased by about $50 \%$, and those of lines at longer wavelengths (around $580 \AA$ ) increased by about $50 \%$. The version 2 calibration included correction factors for the wavelength-dependent instrument degradation partly based on the 15 May 2010 rocket calibration data. Version 3 includes improved degradation corrections using the daily filter and CCD in-flight calibrations and also the addition of the results from another calibration rocket flight in 2011.

For each flare, we have measured the irradiances in a number of lines and obtained light curves, used to then estimate four typical regions, a pre-flare, one during the impulsive phase peak, one during the gradual phase peak (maximum Fe XXI emission), and one later on during the gradual phase decline.

The strongest lines are visible even in C-class flares, as shown in Petkaki et al. (2012). They allow estimates of the electron temperature. However, to estimate densities, large X-class flares are better, since the density-sensitive lines are very weak. We have analysed a few flares, but present in detail here only the results pertaining to one of them, the largest flare observed by both MEGS-A and MEGS-B to date. It occurred on 7 March 2012. It was a large flare (X5.6) with an associated coronal mass ejection (CME). For this flare, we have also considered the impulsive phase peak.

Some of the other flare spectra analysed are shown in the Appendix. They include the largest flare observed by EVE on 9 August 2011, an X6.9 class, and two smaller ones, an M5.4 class on 6 November 2010, and an M2.0 class, on 12 June 2010.

Figure 1 shows the light curves of several EVE lines, ordered by the approximate temperature of formation. It is interesting to note the large increases (up to a factor of 2) in the low transition-region (TR) lines such as O III during the impulsive phase peaks (there were two for this flare). That TR lines show the impulsive phase very well was well-known from observations with the Solar Maximum Mission (SMM) satellite. For example, very good temporal (down to 1s) and spatial correlation between the brightenings of TR lines and hard X-ray emission was found (see, e.g. Cheng et al. 1981; Poland et al. 1982; Woodgate et al. 1983). These enhancements are thought to be due to thermal and nonthermal excitation of the lines in the chromosphere by energetic electrons and particles and the sequential heating and expanding of the chromosphere. What EVE clearly shows (for the first time to our knowledge) is that TR lines formed at higher temperatures show a progressively smoother behaviour, with smaller increases. The chromospheric helium resonances lines also have a smoother behaviour.

The coronal lines formed around 1-2 MK mainly show small decreases during the gradual phase, associated with the CME and the coronal dimmings. Many smaller flares do not show such decreases in the coronal lines, which normally do not show any changes (see Appendix A). The flare lines behave as usual, with the highest temperature lines peaking first, and the lower lines at progressively later times, a product of the cooling (Petkaki et al. 2012).

The dashed lines in Fig. 1 indicate the four regions selected (pre-flare, impulsive phase peak, gradual phase peak, and gradual phase decline). For each region, an average spectrum was obtained. The four spectra are shown superimposed in Fig. 2. Aside from a clear continuum enhancement, it is clear which lines are enhanced during the flare. A summary of the main flare lines is given in Table 1 (we only consider flare lines those formed above $5 \mathrm{MK}$ ). It is also clear that in the vast majority of cases 


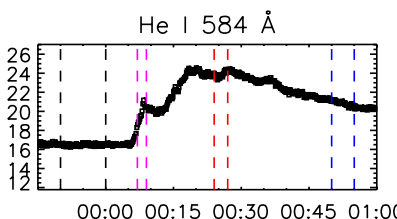

00:00 00:15 00:30 00:45 01:00

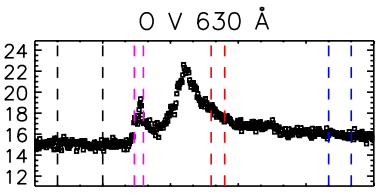

00:00 00:15 00:30 00:45 01:00

Fe XIII $202 \AA$

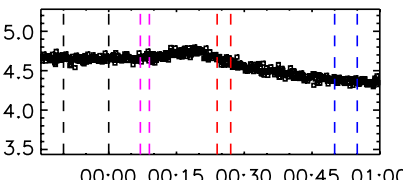

Fe XX $122 \AA$

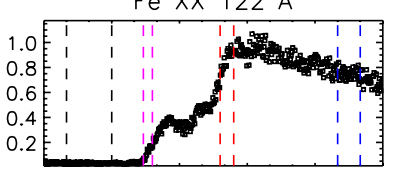

00:00 00:15 00:30 00:45 01:00
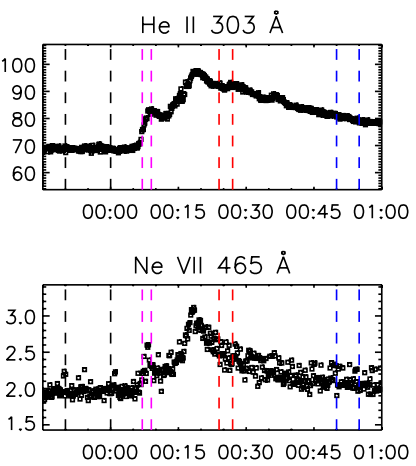

Fe XVI $335 \AA$

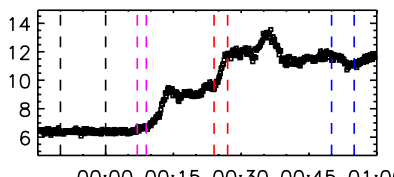

Fe XXI $129 \AA$

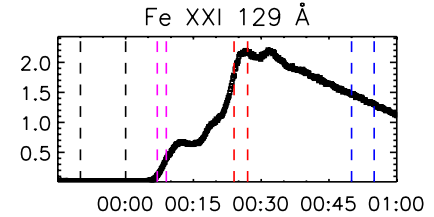

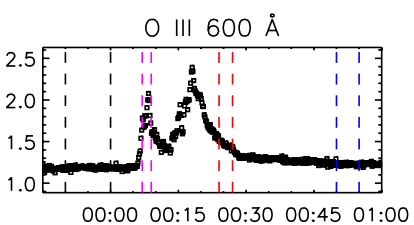

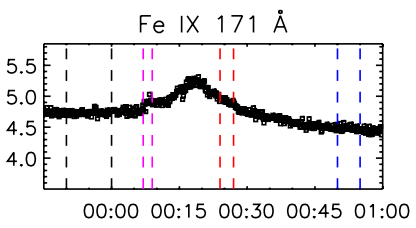

Fe XVIII $94 \AA$

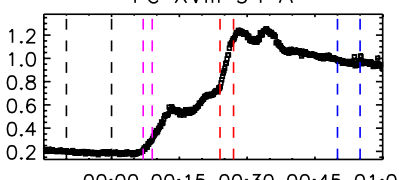

$00: 00 \quad 00: 15 \quad 00: 30 \quad 00: 45 \quad 01: 00$

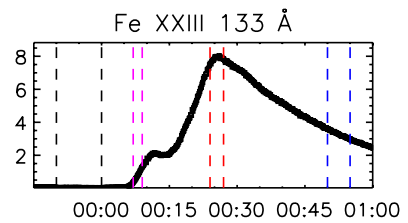

Fig. 1. Light curves of several EVE lines during the X-class flare on 7 March 2012. Irradiances are in $10^{8}$ phot $\mathrm{cm}^{-2} \mathrm{~s}^{-1}$, time in UT. The dashed lines indicate the four regions selected (pre-flare, impulsive phase peak, gradual phase peak, and gradual phase decline).
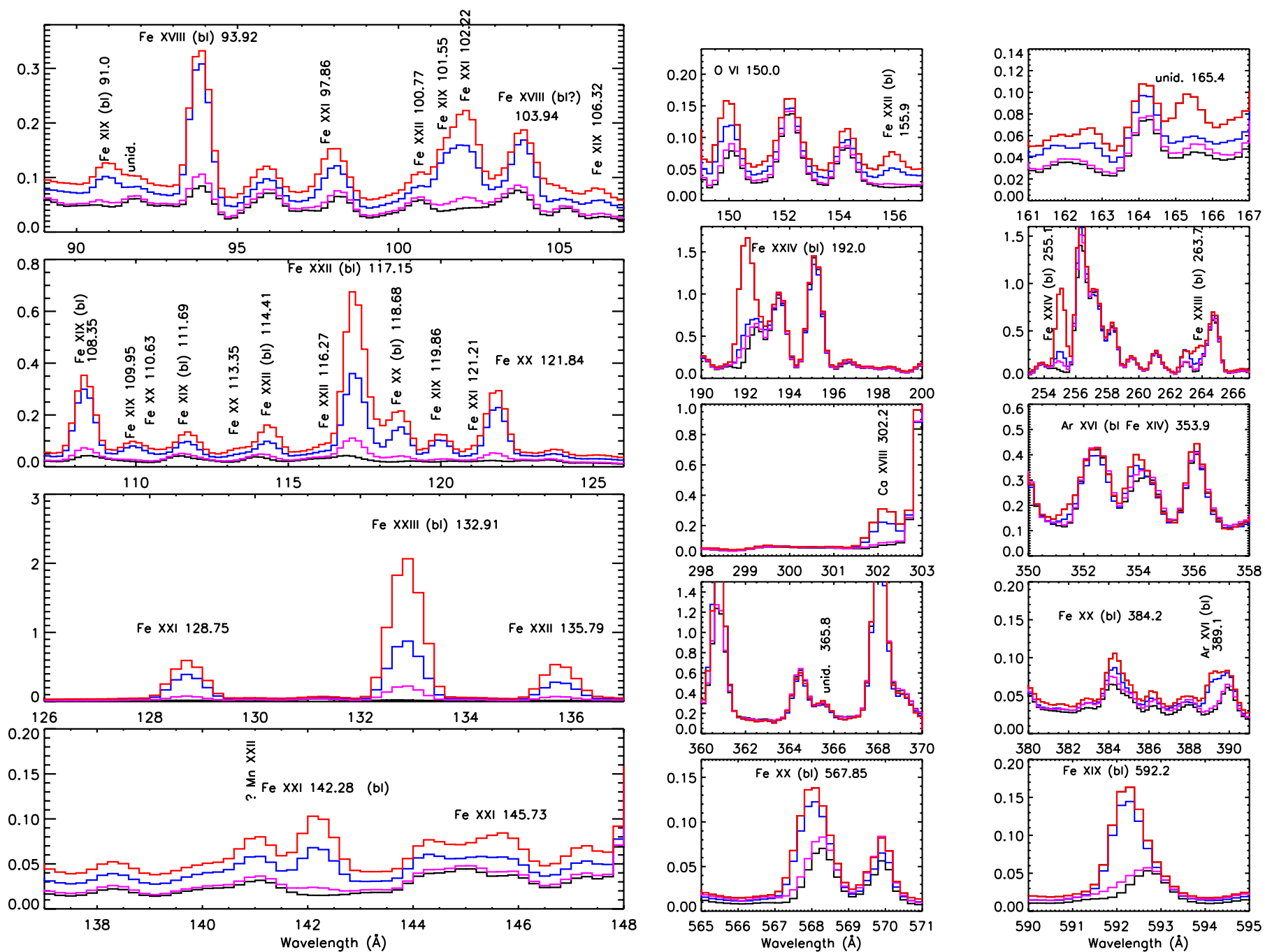

Fig. 2. EVE spectra $\left(10^{8}\right.$ phot $\left.\mathrm{cm}^{-2} \mathrm{~s}^{-1} \mathrm{bin}^{-1}\right)$ during the pre-flare (black), impulsive phase peak (purple), gradual phase peak (red), and gradual phase decline (blue) of the X5.6 class flare on 7 March 2012. The main ions and wavelengths ( $)$ are indicated. 
Table 1. List of the main SDO EVE flare lines.

\begin{tabular}{|c|c|c|c|c|c|}
\hline Ion & $\lambda_{\mathrm{obs}}$ & $\lambda$ & Transition & $T_{\max }$ & Notes \\
\hline ? Ni XXIV & & 88.61 & $2 \mathrm{~s}^{2} 2 \mathrm{p}^{2} \mathrm{P}_{1 / 2}-2 \mathrm{~s} 2 \mathrm{p}^{2}{ }^{2} \mathrm{P}_{1 / 2}$ & 7.2 & very weak \\
\hline $\begin{array}{l}\text { Fe XIX } \\
\text { Fe XXI }\end{array}$ & $\begin{array}{l}91.0 \\
91.0\end{array}$ & $\begin{array}{l}91.013 \\
91.27\end{array}$ & $\begin{array}{l}2 \mathrm{~s}^{2} 2 \mathrm{p}^{4}{ }^{1} \mathrm{D}_{2}-2 \mathrm{~s} 2 \mathrm{p}^{5}{ }^{1} \mathrm{P}_{1} \\
2 \mathrm{~s}^{2} 2 \mathrm{p}^{2}{ }^{3} \mathrm{P}_{0}-2 \mathrm{~s} 2 \mathrm{p}^{3}{ }^{3} \mathrm{~S}_{1}\end{array}$ & $\begin{array}{l}7.0 \\
7.1\end{array}$ & \\
\hline ? Ni XXIII & 92.1 & 91.869 & $2 \mathrm{~s}^{2} 2 \mathrm{p}^{2}{ }^{3} \mathrm{P}_{2}-2 \mathrm{~s} 2 \mathrm{p}^{3}{ }^{3} \mathrm{~s}_{1}$ & 7.2 & \\
\hline $\begin{array}{l}\text { Fe Xx } \\
\text { Fe XVIII }\end{array}$ & $\begin{array}{l}93.8 \\
93.8\end{array}$ & $\begin{array}{l}93.781 \\
93.923\end{array}$ & $\begin{array}{l}2 \mathrm{~s}^{2} 2 \mathrm{p}^{3}{ }^{2} \mathrm{D}_{5 / 2}-2 \mathrm{~s} 2 \mathrm{p}^{4}{ }^{2} \mathrm{P}_{3 / 2} \\
2 \mathrm{~s}^{2} 2 \mathrm{p}^{5}{ }^{2} \mathrm{P}_{3 / 2}-2 \mathrm{~s} 2 \mathrm{p}^{6}{ }^{2} \mathrm{~S}_{1 / 2}\end{array}$ & $\begin{array}{l}7.0 \\
6.9\end{array}$ & bl Fe XIV \\
\hline ? Ni XXI & & 95.86 & $2 \mathrm{~s} 22 \mathrm{p} 43 \mathrm{P} 2-2 \mathrm{~s} 2 \mathrm{p} 53 \mathrm{P} 2$ & 7.1 & \\
\hline $\begin{array}{l}\text { Fe XXI } \\
\text { Fe XVII }\end{array}$ & $\begin{array}{l}97.9 \\
97.9\end{array}$ & $\begin{array}{l}97.864 \\
98.25\end{array}$ & $\begin{array}{l}2 \mathrm{~s}^{2} 2 \mathrm{p}^{2}{ }^{3} \mathrm{P}_{1}-2 \mathrm{~s} 2 \mathrm{p}^{3}{ }^{3} \mathrm{~S}_{1} \\
2 \mathrm{~s}^{2} 2 \mathrm{p}^{5} 3 \mathrm{~s}^{3} \mathrm{P}_{1}-2 \mathrm{p}^{6} 3 \mathrm{~s}^{1} \mathrm{~S}_{0}\end{array}$ & $\begin{array}{l}7.1 \\
6.9\end{array}$ & \\
\hline $\begin{array}{l}\text { Fe XXII } \\
\text { Fe XIX } \\
\text { Fe XXI } \\
\text { Fe XVIII } \\
\text { Fe XIX }\end{array}$ & $\begin{array}{l}100.6 \\
101.5 \\
102.2 \\
103.9 \\
106.1\end{array}$ & $\begin{array}{l}100.775 \\
101.55 \\
102.217 \\
103.948 \\
106.317\end{array}$ & $\begin{array}{l}2 \mathrm{~s}^{2} 2 \mathrm{p}^{2} \mathrm{P}_{1 / 2}-2 \mathrm{~s} 2 \mathrm{p}^{2}{ }^{2} \mathrm{P}_{3 / 2} \\
2 \mathrm{~s}^{2} 2 \mathrm{p}^{4}{ }^{3} \mathrm{P}_{2}-2 \mathrm{~s} 2 \mathrm{p}^{5}{ }^{3} \mathrm{P}_{1} \\
2 \mathrm{~s}^{2} 2 \mathrm{p}^{2}{ }^{3} \mathrm{P}_{2}-2 \mathrm{~s} 2 \mathrm{p}^{3}{ }^{3} \mathrm{~S}_{1} \\
2 \mathrm{~s}^{2} 2 \mathrm{p}^{5}{ }^{2} \mathrm{P}_{1 / 2}-2 \mathrm{~s} 2 \mathrm{p}^{6}{ }^{2} \mathrm{~S}_{1 / 2} \\
2 \mathrm{~s}^{2} 2 \mathrm{p}^{4}{ }^{3} \mathrm{P}_{1}-2 \mathrm{~s} 2 \mathrm{p}^{5}{ }^{3} \mathrm{P}_{0}\end{array}$ & $\begin{array}{l}7.1 \\
7.0 \\
7.1 \\
6.9 \\
7.0\end{array}$ & weak \\
\hline $\begin{array}{l}\text { Fe XXI } \\
\text { Fe XIX }\end{array}$ & $\begin{array}{l}108.3 \\
108.3\end{array}$ & $\begin{array}{l}108.118 \\
108.355\end{array}$ & $\begin{array}{l}2 \mathrm{~s}^{2} 2 \mathrm{p}^{2}{ }^{3} \mathrm{P}_{0}-2 \mathrm{~s} 2 \mathrm{p}^{3}{ }^{3} \mathrm{P}_{1} \\
2 \mathrm{~s}^{2} 2 \mathrm{p}^{4}{ }^{3} \mathrm{P}_{2}-2 \mathrm{~s} 2 \mathrm{p}^{5} \mathrm{C}_{2}\end{array}$ & $\begin{array}{l}7.1 \\
7.0\end{array}$ & $\begin{array}{c}\text { weak } \\
\text { strong }\end{array}$ \\
\hline $\begin{array}{l}\text { Fe XIX } \\
\text { Fe XX }\end{array}$ & $\begin{array}{l}109.9 \\
110.4\end{array}$ & $\begin{array}{l}109.952 \\
110.627\end{array}$ & $\begin{array}{l}2 \mathrm{~s}^{2} 2 \mathrm{p}^{4}{ }^{3} \mathrm{P}_{0}-2 \mathrm{~s} 2 \mathrm{p}^{5}{ }^{3} \mathrm{P}_{1} \\
2 \mathrm{~s}^{2} 2 \mathrm{p}^{3}{ }^{2} \mathrm{D}_{3 / 2}-2 \mathrm{~s} 2 \mathrm{p}^{4}{ }^{2} \mathrm{D}_{3 / 2}\end{array}$ & $\begin{array}{l}7.0 \\
7.0\end{array}$ & weak \\
\hline $\begin{array}{l}\text { Fe XIX } \\
\text { Ni XXIII }\end{array}$ & $\begin{array}{l}111.7 \\
111.7\end{array}$ & $\begin{array}{l}111.695 \\
111.829\end{array}$ & $\begin{array}{l}2 \mathrm{~s}^{2} 2 \mathrm{p}^{4}{ }^{3} \mathrm{P}_{1}-2 \mathrm{~s} 2 \mathrm{p}^{5}{ }^{3} \mathrm{P}_{1} \\
2 \mathrm{~s}^{2} 2 \mathrm{p}^{2}{ }^{3} \mathrm{P}_{0}-2 \mathrm{~s} 2 \mathrm{p}^{3}{ }^{3} \mathrm{D}_{1}\end{array}$ & $\begin{array}{l}7.0 \\
7.2\end{array}$ & $\begin{array}{l}\text { weak } \\
\text { weak }\end{array}$ \\
\hline $\begin{array}{l}\text { Fe Xx } \\
\text { Fe XXII } \\
\text { Fe XXII }\end{array}$ & $\begin{array}{l}113.3 \\
114.4 \\
116.0\end{array}$ & $\begin{array}{l}113.349 \\
114.410 \\
116.268\end{array}$ & $\begin{array}{l}2 \mathrm{~s}^{2} 2 \mathrm{p}^{3}{ }^{2} \mathrm{D}_{5 / 2} 2 \mathrm{~s} 2 \mathrm{p}^{4}{ }^{2} \mathrm{D}_{5 / 2} \\
2 \mathrm{~s}^{2} 2 \mathrm{p}^{2} \mathrm{P}_{3 / 2}-2 \mathrm{~s} 2 \mathrm{p}^{2}{ }^{2} \mathrm{P}_{3 / 2} \\
2 \mathrm{~s}^{2} 2 \mathrm{p}^{2} \mathrm{P}_{3 / 2}-2 \mathrm{~s} 2 \mathrm{p}^{2}{ }^{2} \mathrm{~S}_{1 / 2}\end{array}$ & $\begin{array}{l}7.0 \\
7.1 \\
7.1\end{array}$ & \\
\hline $\begin{array}{l}\text { Fe XXII } \\
\text { Fe XXI }\end{array}$ & $\begin{array}{l}117.2 \\
117.2\end{array}$ & $\begin{array}{l}117.154 \\
117.50\end{array}$ & $\begin{array}{l}2 s^{2} 2 p^{2} \mathrm{P}_{1 / 2}-2 \mathrm{~s} 2 \mathrm{p}^{2}{ }^{2} \mathrm{P}_{1 / 2} \\
2 \mathrm{~s}^{2} 2 \mathrm{p}^{2}{ }^{3} \mathrm{P}_{1}-2 \mathrm{~s} 2 \mathrm{p}^{3}{ }^{3} \mathrm{P}_{1}\end{array}$ & $\begin{array}{l}7.1 \\
7.1\end{array}$ & strong \\
\hline $\begin{array}{l}\mathrm{NixxV} \\
\text { Ni XXII }\end{array}$ & & $\begin{array}{l}117.939 \\
117.918\end{array}$ & $\begin{array}{l}2 \mathrm{~s}^{2}{ }^{1} \mathrm{~s}_{0}-2 \mathrm{~s} 2 \mathrm{p}{ }^{1} \mathrm{P}_{1} \\
2 \mathrm{~s}^{2}{ }^{2} \mathrm{p}^{3}{ }^{4} \mathrm{~S}_{3 / 2}-2 \mathrm{~s} 2 \mathrm{p}^{4}{ }^{4} \mathrm{P}_{5 / 2}\end{array}$ & $\begin{array}{l}7.3 \\
7.0\end{array}$ & \\
\hline $\begin{array}{l}\text { ? Ni XXIV } \\
\text { Fe Xx }\end{array}$ & $\begin{array}{l}118.5 \\
118.5\end{array}$ & $\begin{array}{l}118.474 \\
118.680\end{array}$ & $\begin{array}{l}2 s^{2} 2 p^{2} \mathrm{P}_{1 / 2}-2 \mathrm{~s} 2 \mathrm{p}^{2}{ }^{2} \mathrm{D}_{3 / 2} \\
2 \mathrm{~s}^{2} 2 \mathrm{p}^{3}{ }^{4} \mathrm{~S}_{3 / 2}-2 \mathrm{~s} 2 \mathrm{p}^{4}{ }^{4} \mathrm{P}_{1 / 2}\end{array}$ & $\begin{array}{l}7.1 \\
7.0\end{array}$ & $\begin{array}{c}\text { very weak } \\
\text { strong }\end{array}$ \\
\hline $\begin{array}{l}\text { Fe XIX } \\
\text { Fe XXI } \\
\text { Fe XX } \\
\text { Fe XXI } \\
\text { Fe XXI }\end{array}$ & $\begin{array}{l}120.0 \\
121.3 \\
121.8 \\
123.8 \\
128.7\end{array}$ & $\begin{array}{l}119.983 \\
121.213 \\
121.845 \\
123.831 \\
128.753\end{array}$ & $\begin{array}{l}2 \mathrm{~s}^{2} 2 \mathrm{p}^{4}{ }^{3} \mathrm{P}_{1}-2 \mathrm{~s} 2 \mathrm{p}^{5}{ }^{3} \mathrm{P}_{2} \\
2 \mathrm{~s}^{2} 2 \mathrm{p}^{2} \mathrm{p}^{3} \mathrm{P}_{2}-2 \mathrm{~s} 2 \mathrm{p}^{3} \mathrm{P}_{2} \\
2 \mathrm{~s}^{2} 2 \mathrm{p}^{3}{ }^{4} \mathrm{~S}_{3 / 2}-2 \mathrm{~s} 2 \mathrm{p}^{4}{ }^{4} \mathrm{P}_{3 / 2} \\
2 \mathrm{~s}^{2} 2 \mathrm{p}^{2}{ }^{3} \mathrm{P}_{2}-2 \mathrm{~s} 2 \mathrm{p}^{3}{ }^{3} \mathrm{P}_{1} \\
2 \mathrm{~s}^{2} 2 \mathrm{p}^{2}{ }^{3} \mathrm{P}_{0}-2 \mathrm{~s} 2 \mathrm{p}^{3}{ }^{3} \mathrm{D}_{1}\end{array}$ & $\begin{array}{l}7.0 \\
7.1 \\
7.0 \\
7.1 \\
7.1\end{array}$ & $\begin{array}{l}\text { weak } \\
\text { strong } \\
\text { weak } \\
\text { strong }\end{array}$ \\
\hline $\begin{array}{l}\text { Fe Xx } \\
\text { Fe XXIII }\end{array}$ & $\begin{array}{l}132.9 \\
132.9\end{array}$ & $\begin{array}{l}132.840 \\
132.906\end{array}$ & $\begin{array}{l}2 \mathrm{~s}^{2} 2 \mathrm{p}^{3}{ }^{4} \mathrm{~s}_{3 / 2}-2 \mathrm{~s} 2 \mathrm{p}^{4}{ }^{4} \mathrm{P}_{5 / 2} \\
2 \mathrm{~s}^{2}{ }^{1} \mathrm{~S}_{0}-2 \mathrm{~s} 2 \mathrm{p}^{1} \mathrm{P}_{1}\end{array}$ & $\begin{array}{l}7.0 \\
7.2\end{array}$ & $\begin{array}{l}\text { strong } \\
\text { strong }\end{array}$ \\
\hline $\begin{array}{l}\text { Fe XXII } \\
? \text { Mn XXII }\end{array}$ & 135.8 & $\begin{array}{l}135.791 \\
141.090\end{array}$ & $\begin{array}{l}2 \mathrm{~s}^{2}{ }^{2} \mathrm{p}^{2} \mathrm{P}_{1 / 2}-2 \mathrm{~s} 2 \mathrm{p}^{2}{ }^{2} \mathrm{D}_{3 / 2} \\
2 \mathrm{~s}^{2}{ }^{1} \mathrm{~S}_{0}-2 \mathrm{~s} 2 \mathrm{p}{ }^{1} \mathrm{P}_{1}\end{array}$ & $\begin{array}{l}7.1 \\
7.1\end{array}$ & strong \\
\hline $\begin{array}{l}\text { Fe XXI } \\
\text { Fe XXI }\end{array}$ & $\begin{array}{l}142.2 \\
142.2\end{array}$ & $\begin{array}{l}142.144 \\
142.281\end{array}$ & $\begin{array}{l}2 \mathrm{~s}^{2} 2 \mathrm{p}^{2}{ }^{3} \mathrm{P}_{1}-2 \mathrm{~s} 2 \mathrm{p}^{3}{ }^{3} \mathrm{D}_{2} \\
2 \mathrm{~s}^{2} 2 \mathrm{p}^{2}{ }^{3} \mathrm{P}_{1}-2 \mathrm{~s} 2 \mathrm{p}^{3}{ }^{3} \mathrm{D}_{1}\end{array}$ & $\begin{array}{l}7.1 \\
7.1\end{array}$ & $\begin{array}{l}\text { weak } \\
\text { weak }\end{array}$ \\
\hline $\begin{array}{l}\text { Fe XXI } \\
\text { Fe XXII } \\
\text { ? Ni XXVI }\end{array}$ & $\begin{array}{l}145.6 \\
156.0\end{array}$ & $\begin{array}{l}145.732 \\
156.019 \\
165.377\end{array}$ & $\begin{array}{l}2 s^{2} 2 p^{2}{ }^{3} \mathrm{P}_{2}-2 \mathrm{~s} 2 \mathrm{p}^{3}{ }^{3} \mathrm{D}_{3} \\
2 \mathrm{~s}^{2} 2 \mathrm{p}^{2} \mathrm{P}_{3 / 2}-2 \mathrm{~s} 2 \mathrm{p}^{2}{ }^{2} \mathrm{D}_{5 / 2} \\
1 \mathrm{~s}^{2} 2 \mathrm{~s}^{2} \mathrm{~S}_{1 / 2}-1 \mathrm{~s}^{2} 2 \mathrm{p}^{2} \mathrm{P}_{3 / 2}\end{array}$ & $\begin{array}{l}7.1 \\
7.1 \\
7.4\end{array}$ & $\begin{array}{c}\text { weak } \\
\text { bl }\end{array}$ \\
\hline Fe XXIV & 192.0 & 192.028 & $1 s^{2} 2 s^{2} \mathrm{~S}_{1 / 2}-1 s^{2} 2 \mathrm{p}^{2} \mathrm{P}_{3 / 2}$ & 7.3 & bl \\
\hline Fe XXIV & 255.1 & 255.114 & $1 s^{2} 2 s^{2} S_{1 / 2}-1 s^{2} 2 p^{2} P_{1 / 2}$ & 7.3 & 1 Fe XVII \\
\hline Fe XXIII & 263.7 & 263.765 & $2 \mathrm{~s}^{2}{ }^{1} \mathrm{~S}_{0}-2 \mathrm{~s} 2 \mathrm{p}^{3} \mathrm{P}_{1}$ & 7.2 & bl Sx \\
\hline Ca XVIII & 302.2 & 302.190 & $1 s^{2} 2 s^{2} S_{1 / 2}-1 s^{2} 2 p^{2} P_{3 / 2}$ & 7.0 & bl Ni XIV \\
\hline ArXVI & 353.8 & 353.920 & $1 s^{2} 2 s^{2} S_{1 / 2}-1 s^{2} 2 p^{2} P_{3 / 2}$ & 7.1 & bl Fe XIV \\
\hline Fe XX & 384.3 & 384.210 & $2 s^{2} 2 p^{3}{ }^{4} S_{3 / 2}-2 s^{2} 2 p^{3}{ }^{2} P_{1 / 2}$ & 7.0 & bl CiV \\
\hline Ar XVI & 389.1 & 389.136 & $1 s^{2} 2 s^{2} S_{1 / 2}-1 s^{2} 2 p^{2} P_{1 / 2}$ & 7.0 & bl Fe XVII \\
\hline & 567.8 & 567.867 & $2 s^{2} 2 p^{3}{ }^{4} s_{3 / 2}-2 s^{2} 2 p^{3}{ }^{2} D_{5 / 2}$ & 7.0 & bl Ne V \\
\hline Fe XIX & 592.2 & 592.236 & $2 \mathrm{~s}^{2} 2 \mathrm{p}^{4}{ }^{3} \mathrm{P}_{2}-2 \mathrm{~s}^{2} 2 \mathrm{p}^{4}{ }^{1} \mathrm{D}_{2}$ & 7.0 & bl Fe XII \\
\hline
\end{tabular}

Notes. $\lambda_{\mathrm{obs}}$ and $\lambda(\AA)$ are the measured (by EVE) and experimental wavelengths, $T_{\max }$ (in logarithm) the approximate temperature of formation of the ion in equilibrium; bl means the line is blended.

the increases in the irradiances are not very large. These become much smaller for M- and C-class flares, hence a careful discussion of the pre-flare spectrum is needed, to assess if some contribution to the increase could be caused by lines formed at lower temperatures.
To assess how well the experimental intensities compare with the predicted ones, we have subtracted the pre-flare spectrum from the peak flare spectrum, and obtained line irradiances by removing a continuum emission. We then use the irradiances to produce emissivity ratio curves (introduced in Paper I, Del Zanna et al. 2004). These curves are obtained by dividing the observed intensities of the lines with their predicted emissivity as a function of the electron density, calculated at a fixed temperature, and normalised to 1 . The crossing (or small spreading around 1) of the curves indicates agreement between observed and predicted line intensities. Significant offsets from unity indicate disagreement which could be related to blends in the observed emission lines, to calibration concerns with the observed emission, or to inaccuracies of the atomic emissivities. The emissivity ratio curves relative to the main Fe XIX-Fe XXII EVE lines are shown in Fig. 3.

We now discuss the main flare lines, ion by ion.

\subsection{FeXVIII}

The main atomic data for this ion are from the UK Rmax/APAP work of Witthoeft et al. (2006). The Fe XVIII ion produces two strong decays from the same upper level, at 93.92 and $103.94 \AA$. The first is a much stronger line, so it is normally preferred over the second one. The $93.92 \AA$ line, in quiet Sun (QS) conditions, is blended with several transitions from Fe VIII, Fe IX, Fe X, Fe XIV, as well as an unidentified line. A thorough discussion is presented in Del Zanna (2012a,b). The only known line that can give a small contribution during a flare is a newly identified Fe XIV line (Del Zanna 2012b). During the gradual phase peak of a flare, up to about $10 \%$ of the observed $93.8 \AA$ intensity is due to an Fe XX $93.78 \AA$ line, which is strongly densitysensitive above $10^{11} \mathrm{~cm}^{-3}$ (see below).

In normal QS conditions, EVE observes a strong line around 103.6 $\AA$, which is a blend of many transitions. The brightest line is an Fe IX $103.58 \AA$, followed by the $102.97,103.16,103.89$, and $104.39 \AA$ lines. The first two are partly due to Ne VIII at 102.91 and $103.08 \AA$, while the others are largely unidentified, although there are two significant Fe XIII lines at 103.72 and 103.93 A. During the gradual phase the Fe XVIII $103.94 \AA$ line most likely dominates the blend.

\subsection{FeXIX}

The main atomic data for this ion are from $\mathrm{Gu}$ (2003). The Fe XIX ion produces various lines in the EVE spectral range, with no significant density diagnostics. The $108.35 \AA$ line is the strongest and best for EM analysis, but it is unfortunately blended with the Fe XXI $108.12 \AA$ line and perhaps other lines as well. The intensity of the Fe XXI line is, however, normally small, of the order of $10 \%$, and could, in principle, be estimated via a branching ratio with the $117.50 \AA$ line. However, this line is severely blended (actually it is mostly due to Fe XXII, see below). The best way to estimate the Fe XXI line is, therefore, to use the strong $128.75 \AA$ line, although above $10^{11.5} \mathrm{~cm}^{-3}$ the $108.12 / 128.75 \AA$ ratio is slightly density sensitive. In the QS, EVE observes a line at 108.21 $\AA$, a blend of two partly unidentified transitions observed by M72 at 108.00 and $108.53 \AA$. The first is mostly due to Fe VIII $108.08 \AA$ and several Fe XII transitions, while the second is possibly partly due to a Fe XII transition (Del Zanna 2012b).

The $91.01 \AA$ line is weak and contributes less than half to the observed flare line around 91.0 А. In QS conditions, there are 

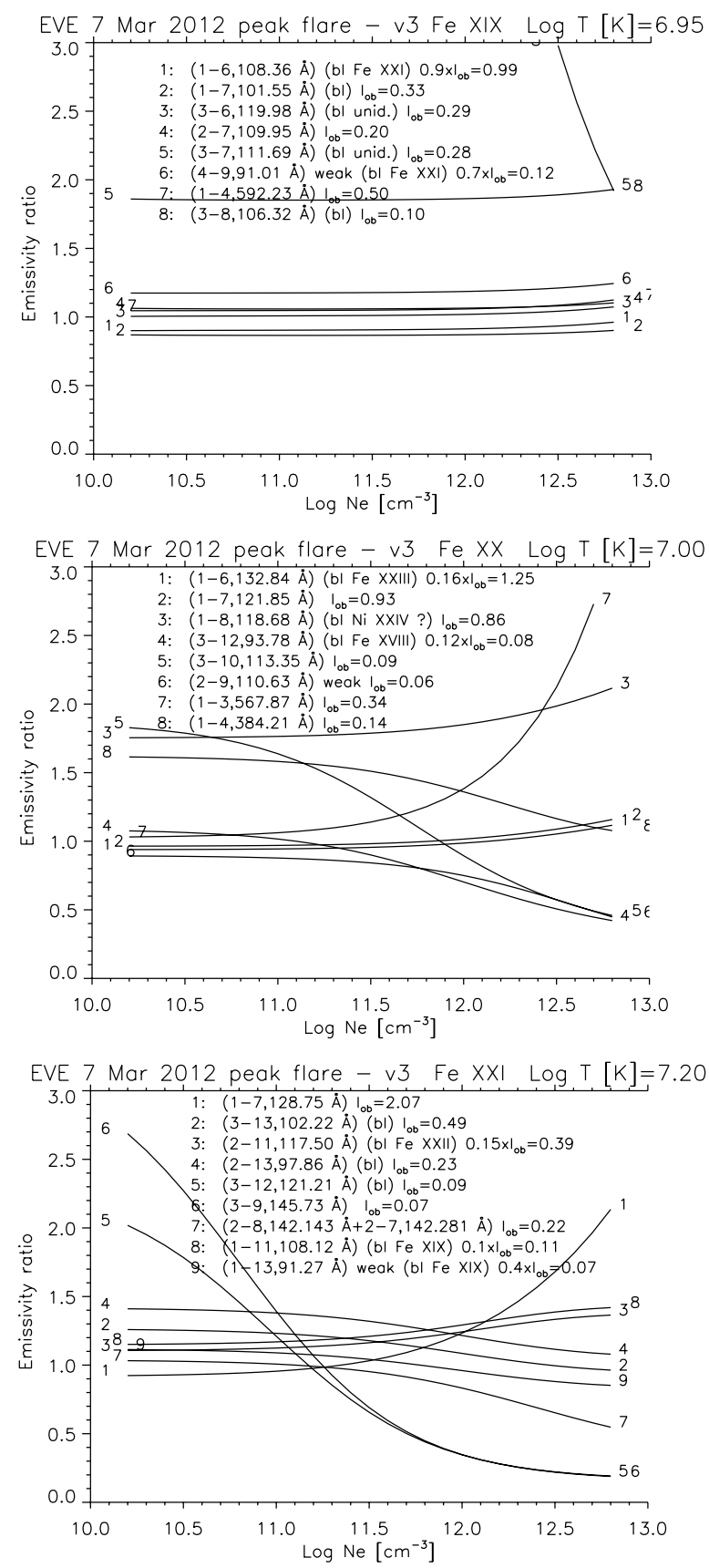

EVE 7 Mar 2012 peak flare - v3 Fe XXII Log $T[K]=7.10$

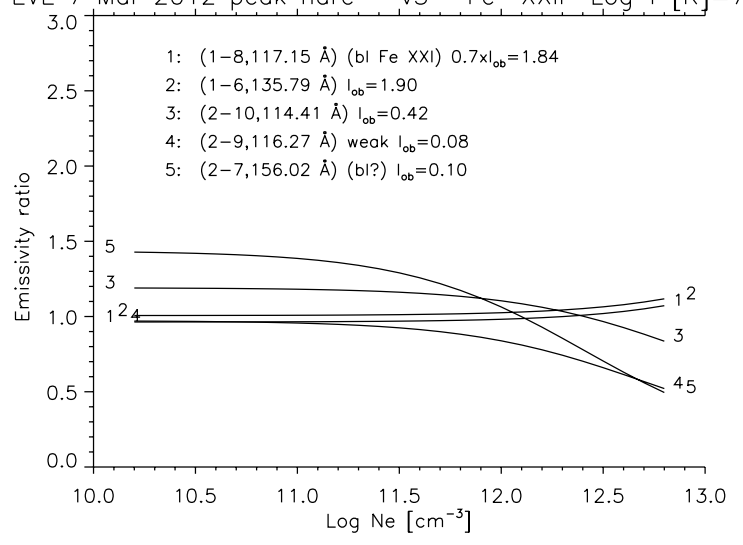

Fig. 3. Emissivity ratio curves relative to the main Fe XIX-Fe XXII EVE lines during the gradual phase peak of the X-class flare on 7 March 2012. $I_{\mathrm{ob}}$ indicates the observed intensity in $10^{8}$ phot $\mathrm{cm}^{-2} \mathrm{~s}^{-1}$, sometimes reduced by the amount indicated; (bl) indicates a blend. several transitions from Fe X, Fe XI, and Fe XII around $91.0 \AA$, as shown in Del Zanna (2012b). In AR conditions, there are also at least contributions from Fe XIII, Fe XVI, and O VII. These transitions increase with density. The Fe XIX $101.55 \AA$ line is located in the blue wing of the stronger Fe XXI 102.22 $\AA$, and so is difficult to measure. In QS conditions, EVE observes a strong line at the same wavelength, $101.55 \AA$. Del Zanna (2012b) identified it as a strong Fe XI transition which increases significantly with density; however, only about half of the observed intensity was accounted for. There is also a possible Fe XIII transition at $101.63 \AA$. The $109.98 \AA$ line is weak and blended with the Fe XX $110.63 \AA$.

The $111.69 \AA$ line is not very strong and is clearly blended in the EVE spectra on its red wing, most probably with the strong resonance Ni XXIII $111.83 \AA$ line. It is difficult to estimate the NiXXIII intensity, given that the other lines are much weaker; however, simple emission measure estimates suggest that this line could have an intensity comparable to the Fe XIX line (the weak line around 92 . $\AA$ could also be due in part to the Ni XXIII $91.87 \AA$ line). In QS conditions, EVE observes a strong line at $111.5 \AA$. Solar spectra show many lines, with the strongest at 111.26 , then at 111.56 and $111.72 \AA$. These lines are still unidentified, although some contribution comes from two Fe IX lines at 111.71 and $111.79 \AA$. Behring et al. (1972) identified the two lines at 111.56 and $111.72 \AA$ with two $\mathrm{Mg}$ VI transitions.

The $119.98 \AA$ line is weak, but appears to be unblended, although a Si XII $119.82 \AA$ line is expected to provide a small contribution. There is a weak line at $106.32 \AA$ which in principle could offer a way to measure densities; however, this line appears to be blended. There is also a weak line at $132.62 \AA$ which is blended with the stronger Fe XXIII and Fe XX lines (see below). Its intensity is approximately $5 \%$ the intensity of the unblended 108.35 A line. Finally, the 592.2 $\AA$ is clearly visible in MEGS-B. With the version 3 EVE data, excellent agreement with the other lines is found (with the version 2 data, the intensity of this line was about $50 \%$ too low). In small flares, this line is blended with an Fe XII 596.6 ̊ identified by Del Zanna \& Mason (2005). In summary, the $108.35 \AA$ line is the recommended line because of its strength (provided that the Fe XXI contribution is removed), together with the much weaker $119.98 \AA$ line.

\subsection{Fexx}

The main atomic data for this ion are from the UK Rmax/APAP work of Witthoeft et al. (2007). The 121.85 $\AA$ is the strongest Fe XX line, after the $132.84 \AA$ line (blended with Fe XXIII, see below). It is the best line to use for EM analyses, but it is clearly blended in its red wing with a weaker line, which is also visible in the QS EVE spectra around 122.5 $\AA$. Behring et al. (1972) reports an unidentified line at $122.72 \AA$. If this line were due to Ne VI, it would increase during the impulsive phase. The $121.85 \AA$ line is blended with a weak Fe XXI line (see below) in its blue wing. The third strongest Fe XX line is at $118.68 \AA$. This line is clearly blended. One possibility in principle would be the Ni XXIV $118.47 \AA$ line. However, this line has a predicted intensity similar to the Ni XXIV line at $88.61 \AA$, a line that is within the noise level, so the $118.47 \AA$ line should also be very weak. There is also a further blend in the blue wing of the $118.68 \AA$ line.

The Fe XX lines offer, in principle, the possibility of measuring electron densities above $10^{11} \mathrm{~cm}^{-3}$, with the well-known $110.63 / 121.85 \AA$ ratio. The $110.63 \AA$ line is however weak, 
and the $113.35 \AA$ line (also weak) is a better density diagnostic, indicating $10^{11.5} \mathrm{~cm}^{-3}$. The $93.78 \AA$ line is blending the stronger Fe XVIII line. This line is strongly density-sensitive above $10^{11} \mathrm{~cm}^{-3}$, and to estimate its intensity the best solution is to consider the ratio with the $110.63 \AA$ line.

Two further lines are observed in MEGS-B, at 384.2 and $567.85 \AA$. With the EVE version 2 data, the $384.2 \AA$ line intensity is in good agreement with theory, but with the version 3 calibration its intensity is about $50 \%$ too high. The $384.2 \AA$ line is blended with two C IV transitions at 384.03 and $384.18 \AA$ and a Mn XV $384.74 \AA$. The C IV lines are expected to have an enhancement during the impulsive phase, but they are intrinsically very weak, so the Fe XX should dominate the blend. With the EVE version 2 data, the intensity of the $567.85 \AA$ line was about $50 \%$ too low. Very good agreement with the other lines is found when the version 3 calibration is used instead. The $567.85 \AA$ line offers a good density diagnostic, although it is blended with a Ne V $568.42 \AA$ line, which most likely will have an enhancement during the impulsive phase. During the gradual phase peak, the Fe XX line should dominate, and indeed the emissivity ratio curve is consistent with densities of the order of $10^{11} \mathrm{~cm}^{-3}$.

\subsection{FeXXI}

The main atomic data for this ion are from Badnell \& Griffin (2001). The Fe XXI ion offers excellent density diagnostics, as described in Mason et al. (1979). By far the strongest transition from this ion is the $128.75 \AA$ line. It is the only clearly observable line that is a decay to the ground state. In the MH73 quiet Sun spectra there are only few weak lines at 128.49, 128.69, 128.92, and $129.22 \AA$, so the Fe XXI line should be relatively free of blends. The 102.22 $\AA$ line is the second strongest line, but with an intensity only about $30 \%$ the $128.75 \AA$ line. In the QS spectra, there is a relatively strong line at $102.12 \AA$, and a weaker one at $102.44 \AA$. There is a host of Fe X, Fe XI, Fe XVI, and $\mathrm{O}$ VIII lines at these wavelengths, which partly account for the observed lines (Del Zanna 2012b).

Above $10^{11.5} \mathrm{~cm}^{-3}$, the $108.12 / 128.75 \AA$ ratio becomes slightly density sensitive; however, the main diagnostic lines are the 121.21 and $145.73 \AA$ lines, although these two are very weak. The first is in the blue wing of the stronger Fe XX $121.84 \AA$ line in flare spectra. In the MH73 quiet Sun spectrum, there are two weak lines at 121.13 and $121.41 \AA$; the second is possibly due to a $3 d-4 p$ Fe XI transition. Del Zanna (2012b) suggested a possible different identification for this line, but if the $121.41 \AA$ line were the Fe XI transition, then it would increase at high densities. The second line at $145.73 \AA$ should provide the best density diagnostic for EVE, according to Milligan et al. (2012b). This line is however clearly blended on the blue side in EVE spectra. In quiet Sun MH73 spectra, there is a strong line at the same wavelength, $145.73 \AA$, plus two weak lines at 145.59 and $146.11 \AA$. The first line is probably due to a Ni X transition at $145.733 \AA$, as will be discussed in a separate paper, while the other two are unidentified. We note that Kastner et al. (1974) reported a wavelength of $145.66 \pm 0.05 \AA$, while Sugar \& Rowan (1995) measured $145.733 \AA$, but noted that the Fe XXI line was blended with Ni X. One additional problem with the $145.73 \AA$ line is that this line does not always vary with time as an Fe XXI line.

There is also a self-blend of Fe XXI lines at 142.14 and $142.28 \AA$, but the ratio with the $128.75 \AA$ line is only sensitive at densities greater than $10^{12} \mathrm{~cm}^{-3}$, as shown in Fig. 3 . The self-blend is located in a region where in the QS there are only weak lines. In the MH73 spectrum, we measured three lines at $141.85,142.23$, and $142.65 \AA$, the last one due to Fe X. Behring et al. (1972) reports instead a line at $142.02 \AA$. The other Fe XXI lines are either blended or not useful to measure densities. The 117.27 is blended with the much stronger Fe XXII 117.15 line. The $97.87 \AA$ is very weak and possibly blended with an Fe XVII line. There is a weak Fe XXI line at $97.86 \AA$ that is strongly density-sensitive, however EVE spectra show the presence of a broad line around $98.0 \AA$, which behaves like the cooler Fe XVIII lines. Del Zanna \& Ishikawa (2009) identified an Fe XVII line at $98.25 \AA$. It is difficult to assess the intensity of this line, given the lack of clearly resolved Fe XVII lines.

In summary, the best diagnostic is the $145.73 / 128.75 \AA$ ratio, considering the diffculty in measuring the $121.21 \AA$ line. The Fe XXI lines are sensitive to a wide range of densities, and indicate a value of about $10^{11.2} \mathrm{~cm}^{-3}$. However, as in previous cases, all the weak lines are blended with unidentified lines, hence any densities obtained should be regarded as upper limits. Despite that, the measured densities are somewhat small for such a large flare. For EM analysis, the best line is the $128.75 \AA$, although as Fig. 3 shows, this line has an emissivity that decreases above $10^{11.5} \mathrm{~cm}^{-3}$, hence such densities (or higher) should be taken into account when calculating the line emissivity for EM analyses, for example.

\subsection{Fe $x x I I$}

The main atomic data for this ion are from Badnell et al. (2001). The Fe XXII ion has two strong decays to the ground, at 117.15 and $135.79 \AA$. The first line is blended in flare spectra with Fe XXI. In its red wing, the line is expected to be blended with the Ni XXV $117.94 \AA$ resonance line. The Fe XXII $135.79 \AA$ line is relatively free of blends because it falls in a spectral region where there are no strong lines in QS spectra. The Fe XXII $135.79 \AA$ is, therefore, the recommended line for EM analysis.

Above $10^{11.5} \mathrm{~cm}^{-3}$, the $114.41 \AA$ line becomes visible and in principle could provide a way to measure densities in combination with the $135.79 \AA$ line, although the sensitivity is not as good as in the Fe XXI case. Also, the 114.41 $\AA$ line is clearly blended on its blue side with a line around $113.8 \AA$ that increases its intensity during flares. Mason et al. (1984) identified the $156.0 \AA$ line as due to Fe XXII. This line would be a better density diagnostic, but appears to be blended, because it provides a very high density. In QS spectra, there are two wellknown strong Fe IX 3d-4f transitions (see O'Dwyer et al. 2012 for a list and the relevant atomic data), at 113.79 and $114.02 \AA$ (blended with an Fe X 114.08, (Del Zanna 2012b)), plus a very weak one at $114.11 \AA$. However, at flare densities of $10^{11} \mathrm{~cm}^{-3}$, the intensity of this last line increases, becoming about $20 \%$ the intensity of the other two lines.

\subsection{FeXXIII}

The main atomic data for this ion are from Chidichimo et al. (2005). The Fe XXIII 132.906 $\AA$ resonance line is blended with the Fe XX $132.84 \AA$ transition, the strongest line from Fe XX. During the gradual phase decline, the contribution of the Fe XX to the blend increases. The Fe XX $132.84 \AA$ line is a straight decay to the ground state, and its intensity can be estimated from the intensity of the other decay to the ground at $121.84 \AA$. This ratio is not density sensitive, and its value mainly depends on the accuracy of the excitation cross sections for the two upper levels, so the Fe XXIII can be deblended quite accurately. 
The Fe XXIII intercombination line at $263.7 \AA$ is observed by MEGS-A. This line, at the EVE resolution, is close to the strong Fe XVI $263.0 \AA$ and Fe XIV $264.8 \AA$ lines, and it is difficult to fit. The Fe XXIII 263.7 ^ line is blended with S X 264.2 $\AA$, a coronal line not expected to vary significantly during the flare. The ratio of the resonance and intercombination lines is, in principle, an excellent temperature diagnostics, as discussed in Del Zanna et al. (2005); however, the temperatures obtained from this ratio are too low. With the EVE version 2 calibration, a large discrepancy in the observed vs. predicted $132.9 / 263.7 \AA$ ratio is present. The new version 3 calibration reduces the intensity of the resonance $132.9 \AA$ line by over $30 \%$. Agreement with theory would be obtained by further reducing the $263.7 \AA$ line by about $30 \%$. It is, therefore, recommended that the resonance $132.9 \AA$ line be used for emission measure analysis.

\subsection{FeXXIV}

The EVE instrument observes the important Fe XXIV 192 and $255.1 \AA$ doublet. These lines are clearly visible only in large flares, because they are normally severely blended, as discussed in Del Zanna et al. (2011). For large flares, the Fe XXIV is expected to dominate. The CaXVII 192.9 $\AA$ resonance line is on the blue-wing of the Fe XXIV $192 \AA$ and has been removed by fitting. The $192 \AA$ line also has residual contamination from Fe XI and $\mathrm{OV}$, but it is expected to be negligible for large flares during the gradual phase peak. The $255.1 \AA$ line is blended with various transitions, the main one being Fe XVII at $254.8 \AA$. The Fe XVII $254.8 \AA$ is, however, expected to be weak since the Fe XVII $204.7 \AA$ is not normally visible. These two lines are the strongest Fe XVII lines in the EVE spectral range (Del Zanna \& Ishikawa 2009).

The CHIANTI atomic data for this ion are partly from Berrington \& Tully (1997). Liang \& Badnell (2011) recently performed a larger R-matrix calculation for this ion, as part of the APAP-network work. Here we have used the Liang \& Badnell (2011) data, noting however that for the doublet little differences in the absolute and relative intensities are present when compared to the previous ion model. In fact, the CHIANTI Fe XXIV 192/255.1 $\AA$ predicted ratio is 1.85 (photons) while the Liang \& Badnell (2011) ratio is 1.9.

The EVE version 3 calibration increases the intensities of these lines by about 20\%. The observed ratio for the 7 Mar. 2012 gradual phase peak is 1.5 with version 3 and 1.6 with version 2 , i.e. not far from the expected ratio (assuming optically thin emission). The ratio can be brought into agreement by reducing the $255 \AA$ irradiance by $30 \%$. It is recommended that the stronger $192 \AA$ line be used for emission measure analysis.

\subsection{Other lines}

There are a number of Fe XVII lines that could, in principle, be observed, as discussed in Del Zanna \& Ishikawa (2009); however, they all turn out to be too weak to be observed with EVE. The resonance line from Ca XVIII at $302.2 \AA$ is clearly observed in the blue-wing of the He II $304 \AA$. It is blended with a Ni XIV 302.27 $\AA$. However, during the gradual phase peak of flares, the Ca XVIII line is expected to dominate the blend.

There are two ArXVI lines that can be used to estimate the argon/iron abundance during flares. The $353.92 \AA$ line is blended with a density-sensitive Fe XIV $353.83 \AA$ line, and the $389.14 \AA$ is blended with an Fe XVII $389.11 \AA$ line

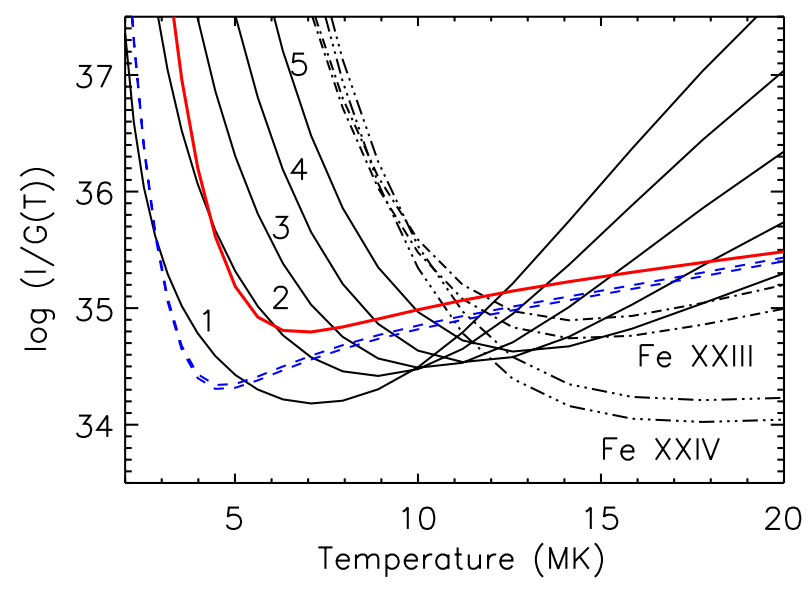

Fig. 4. EM loci curves for the peak flare spectrum and a selection of lines. Iron lines from Fe XVIII (1), Fe XIX (2), Fe XX (3), Fe XXI (4), and Fe XXII (5) are solid, the two Fe XXIII lines dot-dash, and the two Fe XXIV lines triple dot-dash. The Ca XVIII $302.2 \AA$ line is thick solid red. The two Ar XVI lines are dashed blue.

(Del Zanna \& Ishikawa 2009), and on the red wing by another line, possibly Cr XIV at 389.81 . For the gradual phase peak, the increases in both lines are, however, expected to be mainly due to Ar XVI.

The observed intensity of the NiXXVI resonance line at $165.37 \AA$ is stronger than predicted from the EM modelling, assuming that the Fe XXIV emission is correct (see below). This could be due to a blending or to the presence of a hotter component. Finally, there are still several weak unidentified flare lines in the EVE spectra.

\subsection{Temperatures, emission measures, and abundances}

In order to have an estimate of the temperature, EM, and relative abundances for the gradual phase peak, we start by considering the EM loci curves (see Del Zanna et al. 2002 for an explanation of the method). They have been obtained with the Asplund et al. (2009) photospheric abundances, and are shown in Fig. 4. First, it is interesting to notice that the curves relative to Fe XVIII-Fe XXIV all intersect around $12 \mathrm{MK}$, with the exception of the two Fe XXIII lines, shown as dot-dash. This would be consistent with a near-isothermal plasma at $12 \mathrm{MK}$. The discrepancy between the Fe XXIII and Fe XXIV lines was quite significant when the version 2 data were used. With the version 3 calibration, there is only a $30 \%$ disagreement between the two resonance lines (Fe XXIV 192.0 $\AA$ and Fe XXIII 132.9 $\AA$ ).

Second, we note that the curves pertaining to the Ca XVIII 302.2 $\AA$ resonance line and the two Ar XVI lines are in good agreement (within a relative $30 \%$ ) with those from the iron ions, indicating photospheric abundances. This is an important result. We note that argon is a high first ionisation potential (FIP) element, while iron is a low FIP, so the iron/argon relative abundance can be used as a proxy to estimate the elemental abundances, one of the many unsettled issues in solar flare physics.

The discrepancy in the Fe XXIII lines is reflected in the isothermal temperatures that can be obtained from EVE line ratios. As Fig. 4 shows, if the Fe XXIII lines are used in conjunction with Fe XVIII-Fe XXII lines, they provide higher temperatures (where the curves cross) than those obtained with all the other lines. If the Fe XXIV and Fe XXIII lines are used, lower temperatures are found. 


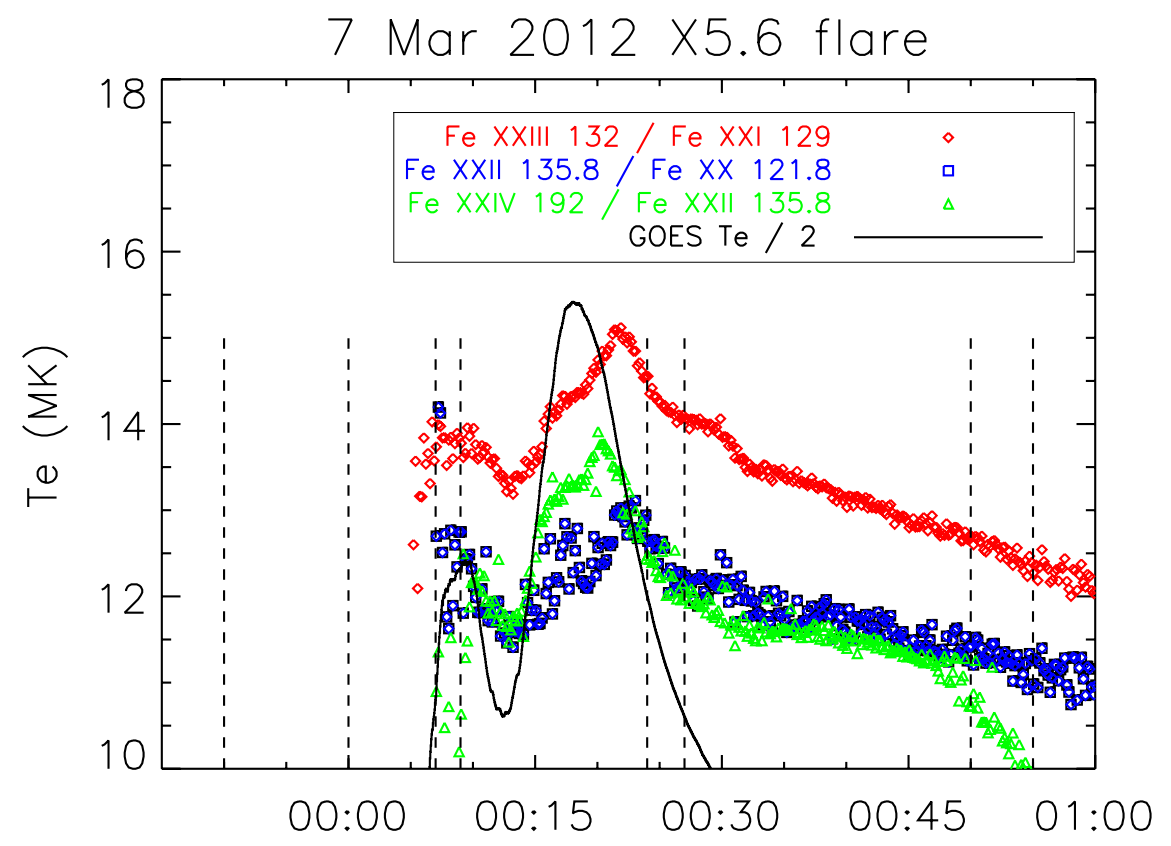

Fig. 5. Isothermal temperatures for the $X$-class flare on 7 March 2012, obtained from three EVE line ratios and from GOES (scaled by a factor of two).
To illustrate that these discrepancies do not occur only during the gradual phase peak, we have calculated the isothermal temperature for a few lines ratios. We have subtracted the preflare irradiances before taking the ratios, and calculated the theoretical ratios assuming ionization equilibrium as usual. The results for three line ratios are shown in Fig. 5. If the Fe XXIII and Fe XXI lines are considered, for example, the peak isothermal temperature reaches $15 \mathrm{MK}$. However, if Fe XXII and Fe XX lines are considered (for example), the peak isothermal temperature is slightly lower, about 13 MK. The Fe XXIV vs. Fe XX ratio indicates reasonable agreement with the Fe XXII vs. Fe XX ratio.

We note that isothermal temperatures obtained from the GOES X-ray fluxes are much higher, reaching temperatures above $20 \mathrm{MK}$. Figure 5 shows the isothermal temperatures obtained from GOES using CHIANTI v.7 atomic data and photospheric abundances (White et al. 2005), scaled down by a factor of two.

We also note that higher temperatures would be expected on the basis of the Feldman et al. (1996) study, although it appears (H. Hudson, priv. comm.) that flares of this last solar maximum might have lower temperatures than those of previous cycles. The other flares we analysed show a similar behaviour, with the differences between the EVE and GOES isothermal temperatures decreasing for smaller flares. Good agreement between the EVE temperatures and those from GOES was indeed found for a C-class flare (Petkaki et al. 2012). The GOES satellites are broad-band instruments, so they are expected to be dominated by continuum emission, especially in large flares where it is known that continuum emission increases significantly. It is therefore possible that the GOES temperatures are higher because of the continuum. Given the nature of large flares, where several different structures contribute to the emission, it would be natural to expect a multi-thermal distribution of temperatures, a possibility that will be explored in a separate paper, where line and continuum emission of different flares will be combined.

\section{Conclusions}

The calibrated EVE irradiances have allowed us, for the first time since OSO-5, to assess the atomic data using solar observations. Good overall agreement between predicted and observed line ratios is found once all the blends are taken into account. This provides confidence in the reliability of the atomic data. However, the benchmark and comparison with quiet Sun spectra has clearly shown that more work is needed to provide atomic data and identifications in the soft $\mathrm{X}$-rays, given that a number of lines that potentially blend the EVE flare lines are still unidentified. Despite this, we have clearly shown that EVE has a great potential to measure electron temperatures and emission measures at very high temporal cadence. The lines recommended for temperature and EM analyses are: Fe XVIII (deblended) $93.92 \AA$ A, Fe XIX (deblended) $108.35 \AA$, Fe XX $121.85 \AA$, Fe XXI $128.75 \AA$ (below $10^{11.5} \mathrm{~cm}^{-3}$ ), Fe XXII $135.79 \AA$, Fe XXIII (deblended) $132.91 \AA$, and Fe XXIV $192.0 \AA$.

Measurements of electron densities are more difficult, but there are several potential diagnostic possibilities, once blending and calibration issues are sorted out. Currently, the best diagnostic ratio is the Fe XXI 145.73/128.75 $\AA$, although measurements are limited to large flares.

We have also found that it is possible to estimate the relative argon/iron and calcium/iron abundances during the gradual phase peak of a flare, using the Ar XVI and Ca XVIII lines. These are important diagnostics. The X5.6 flare showed photospheric abundances, and relatively low electron densities and temperatures.

The new version 3 EVE calibration removed all the main discrepancies between observation and theory, although a few still remain and will need to be investigated further. Understanding the discrepancies between the EVE and GOES isothermal temperatures is beyond the scope of this paper, and a full analysis is left to a future paper. During the impulsive phase, departures from ionization equilibrium could occur. However, during the gradual phase, when densities are high, equilibrium should hold, as commonly assumed in most analyses. It is therefore possible that the GOES isothermal temperatures are biased by the continuum emission, or that additional high-temperature emission is observed by GOES and not EVE.

Above all, the great potential of the EVE spectra is the possibility of measuring with high temporal cadence the evolution 
of lines formed at different temperatures. We have clearly seen that lines normally formed in the quiet Sun in the low transition region such as $\mathrm{O}$ III are the best to study the impulsive phase.

Acknowledgements. We thank the anonymous referee for useful suggestions on how to improve the paper. G.D.Z. acknowledges support from STFC. The work of the UK APAP Network was funded by the UK STFC under grant No. PP/E001254/1 with the University of Strathclyde. CHIANTI is a collaborative project involving researchers at the Universities of: Cambridge (UK), George Mason, Michigan (USA). T.N.W. acknowledges support from NASA under NASA contract NAS5-02140 to the University of Colorado. The SDO EVE calibration is an on-going effort of many people, with special thanks to Frank Eparvier, Andrew Jones, and Don Woodraska at the University of Colorado.

\section{References}

Asplund, M., Grevesse, N., Sauval, A. J., \& Scott, P. 2009, ARA\&A, 47, 481 Badnell, N. R., \& Griffin, D. C. 2001, J. Phys. B Atom. Mol. Phys., 34, 681 Badnell, N. R., Griffin, D. C., \& Mitnik, D. M. 2001, J. Phys. B Atom. Mol. Phys., 34, 5071

Behring, W. E., Cohen, L., \& Feldman, U. 1972, ApJ, 175, 493

Berrington, K. A., \& Tully, J. A. 1997, A\&AS, 126, 105

Chamberlin, P. C., Milligan, R. O., \& Woods, T. N. 2012, Sol. Phys., 279, 23

Cheng, C.-C., Tandberg-Hanssen, E., Bruner, E. C., et al. 1981, ApJ, 248, L39

Chidichimo, M. C., Del Zanna, G., Mason, H. E., et al. 2005, A\&A, 430, 331

Del Zanna, G. 1995, Ph.D. Thesis, Univ. of Florence, Italy

Del Zanna, G. 2012a, ApJ, submitted

Del Zanna, G. 2012b, A\&A, 546, A97

Del Zanna, G. 2012c, A\&A, 537, A38

Del Zanna, G., \& Ishikawa, Y. 2009, A\&A, 508, 1517

Del Zanna, G., \& Mason, H. E. 2005, A\&A, 433, 731

Del Zanna, G., \& Storey, P. J. 2012, A\&A, 543, A144

Del Zanna, G., \& Storey, P. J. 2013, A\&A, 549, A42

Del Zanna, G., Landini, M., \& Mason, H. E. 2002, A\&A, 385, 968

Del Zanna, G., Berrington, K. A., \& Mason, H. E. 2004, A\&A, 422, 731

Del Zanna, G., Chidichimo, M. C., \& Mason, H. E. 2005, A\&A, 432, 1137

Del Zanna, G., Mitra-Kraev, U., Bradshaw, S. J., Mason, H. E., \& Asai, A. 2011, A\&A, 526, A1

Del Zanna, G., Storey, P. J., Badnell, N. R., \& Mason, H. E. 2012a, A\&A, 543, A139

Del Zanna, G., Storey, P. J., Badnell, N. R., \& Mason, H. E. 2012b, A\&A, 541, A90
Edlén, B. 1937, Z. Astrophys., 104, 407

Fawcett, B. C., \& Cowan, R. D. 1975, MNRAS, 171, 1

Fawcett, B. C., Kononov, E. Y., Hayes, R. W., \& Cowan, R. D. 1972, J. Phys. B Atom. Mol. Phys., 5, 1255

Feldman, U., Doschek, G. A., Behring, W. E., \& Phillips, K. J. H. 1996, ApJ, 460, 1034

Gu, M. F. 2003, ApJ, 582, 1241

Hudson, H. S., Woods, T. N., Chamberlin, P. C., et al. 2011, Sol. Phys., 273, 69

Kastner, S. O., Neupert, W. M., \& Swartz, M. 1974, ApJ, 191, 261

Kononov, E. Y., Koshelev, K. N., Podobedova, L. I., Chekalin, S. V., \& Churilov, S. S. 1976, J. Phys. B Atom. Mol. Phys., 9, 565

Landi, E., Young, P. R., Dere, K. P., Del Zanna, G., \& Mason, H. E. 2013, ApJ, 763,86

Lemen, J. R., Title, A. M., Akin, D. J., et al. 2012, Sol. Phys., 275, 17

Liang, G. Y., \& Badnell, N. R. 2011, A\&A, 528, A69

Liang, G. Y., Whiteford, A. D., \& Badnell, N. R. 2009, A\&A, 500, 1263

Liang, G. Y., Badnell, N. R., Crespo López-Urrutia, J. R., et al. 2010, ApJS, 190, 322

Malinovsky, L., \& Heroux, M. 1973, ApJ, 181, 1009

Manson, J. E. 1972, Sol. Phys., 27, 107

Mason, H. E., Doschek, G. A., Feldman, U., \& Bhatia, A. K. 1979, A\&A, 73, 74

Mason, H. E., Bhatia, A. K., Neupert, W. M., Swartz, M., \& Kastner, S. O. 1984, Sol. Phys., 92, 199

Mewe, R., Raassen, A. J. J., Drake, J. J., et al. 2001, A\&A, 368, 888

Milligan, R. O., Chamberlin, P. C., Hudson, H. S., et al. 2012a, ApJ, 748, L14

Milligan, R. O., Kennedy, M. B., Mathioudakis, M., \& Keenan, F. P. 2012b, ApJ, 755, L16

Monsignori Fossi, B. C., Landini, M., Del Zanna, G., \& Bowyer, S. 1996, ApJ, 466,427

O’Dwyer, B., Del Zanna, G., Badnell, N. R., Mason, H. E., \& Storey, P. J. 2012, A\&A, 537, A22

Petkaki, P., Del Zanna, G., Mason, H. E., \& Bradshaw, S. 2012, A\&A, 547, A25

Poland, A. I., Frost, K. J., Woodgate, B. E., et al. 1982, Sol. Phys., 78, 201

Stratton, B. C., Moos, H. W., \& Finkenthal, M. 1984, ApJ, 279, L31

Sugar, J., \& Rowan, W. L. 1995, Opt. Soc. Am. J. B Opt. Phys., 12, 1403

White, S. M., Thomas, R. J., \& Schwartz, R. A. 2005, Sol. Phys., 227, 231

Witthoeft, M. C., Badnell, N. R., Del Zanna, G., Berrington, K. A., \& Pelan, J. C. 2006, A\&A, 446, 361

Witthoeft, M. C., Del Zanna, G., \& Badnell, N. R. 2007, A\&A, 466, 763

Woodgate, B. E., Shine, R. A., Poland, A. I., \& Orwig, L. E. 1983, ApJ, 265, 530

Woods, T. N., Eparvier, F. G., Hock, R., et al. 2012, Sol. Phys., 275, 115

Pages 10 to 12 are available in the electronic edition of the journal at http://www . aanda. org 

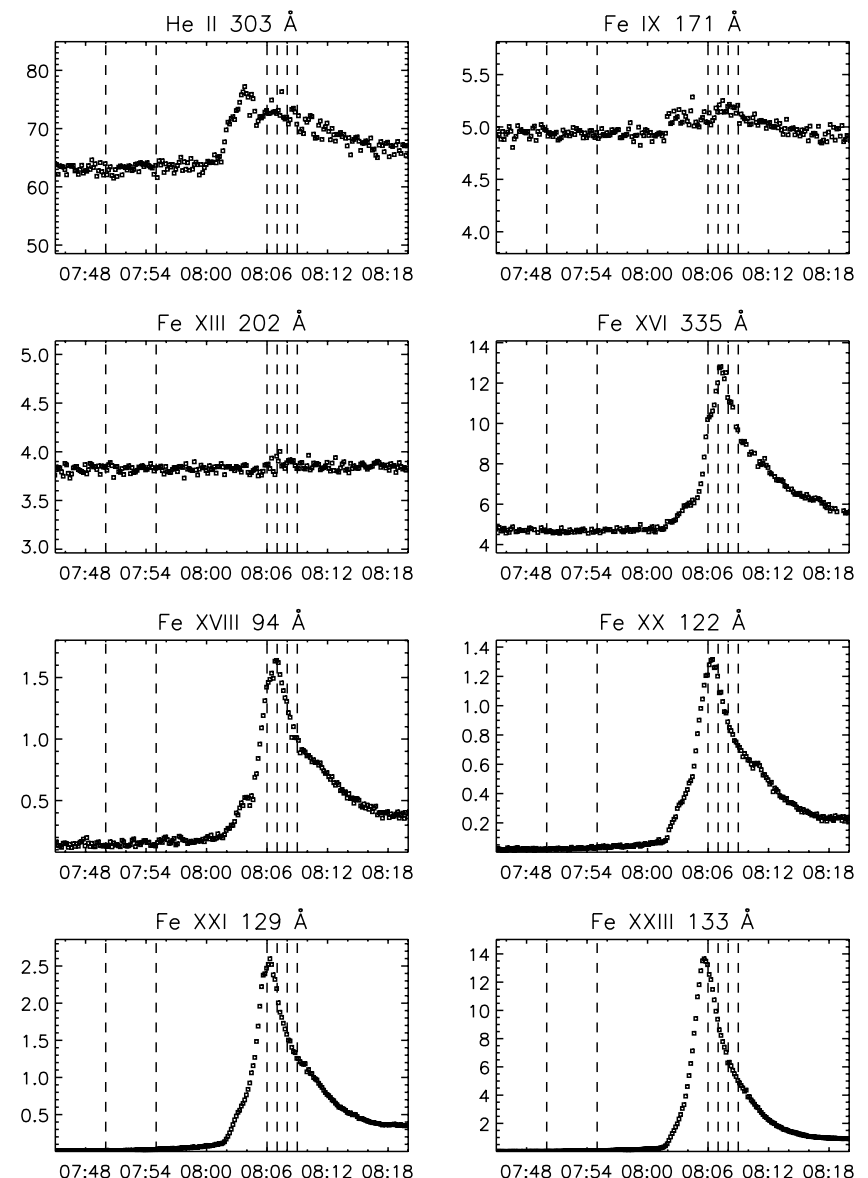

Fig. A.1. Light curves of several EVE lines during the X6.9 class flare on 9 August 2011. Irradiances are in $10^{8}$ phot $\mathrm{cm}^{-2} \mathrm{~s}^{-1}$, time in UT. The dashed lines indicate the three regions selected (pre-flare, gradual phase peak, and gradual phase decline).

\section{Appendix A: Other flares}

On 9 August 2011, the Sun produced the largest flare observed by EVE, an X6.9 class; MEGS-B did not observe this flare. Figure A.1 shows the light curves, while Fig. A.2 shows the spectra. Figures A.3 and A.4 show the light curves and the
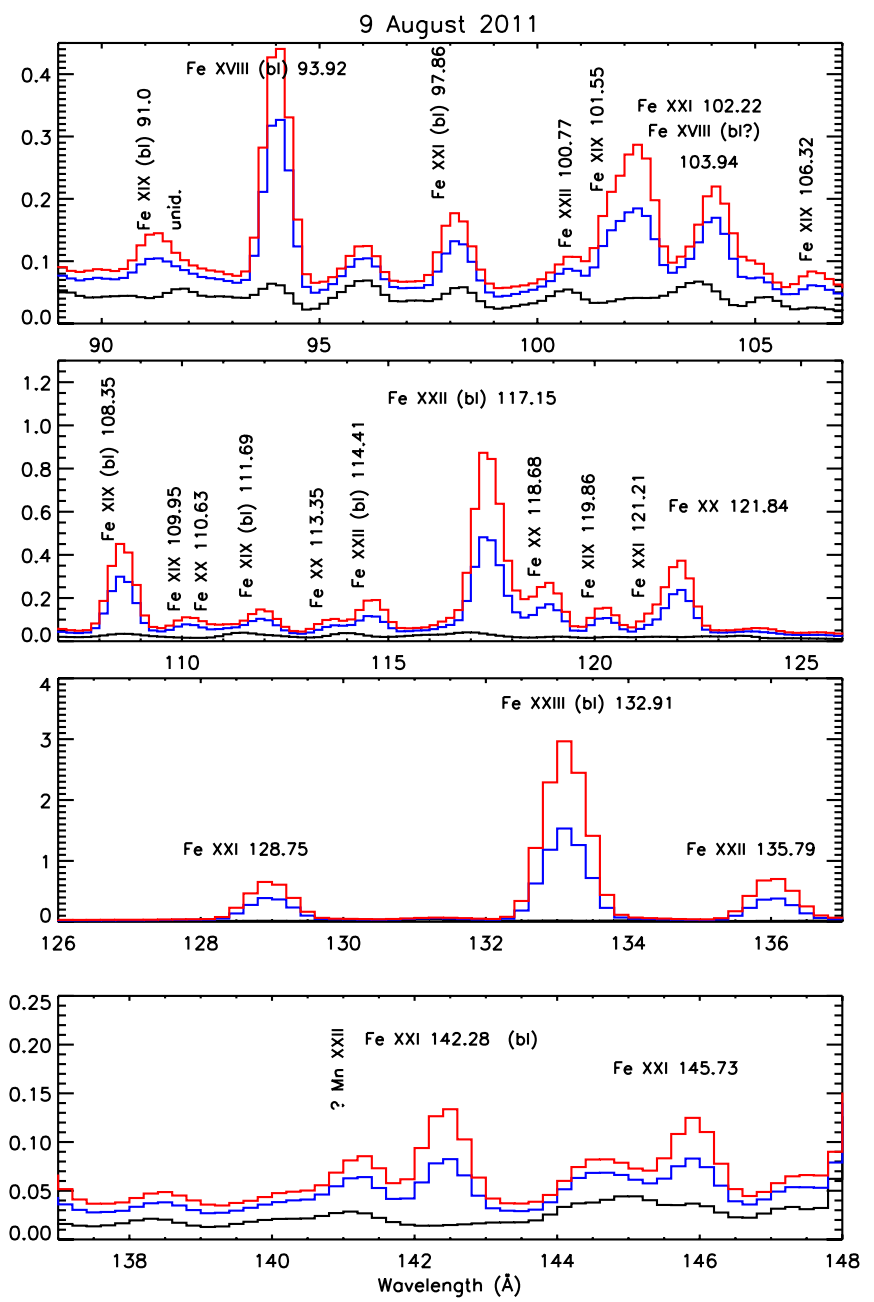

Fig. A.2. EVE spectra during the pre-flare (black), gradual phase peak (red), and gradual phase decline (blue) of the X6.9 class flare on 9 August 2011.

spectra of the 6 Nov 2010 M5.4 class flare. Figures A.5 and A.6 show the light curves and the spectra of the smaller M2.0 class flare on 12 June 2010. 
G. Del Zanna and T. N. Woods: Spectral diagnostics with the SDO EVE flare lines
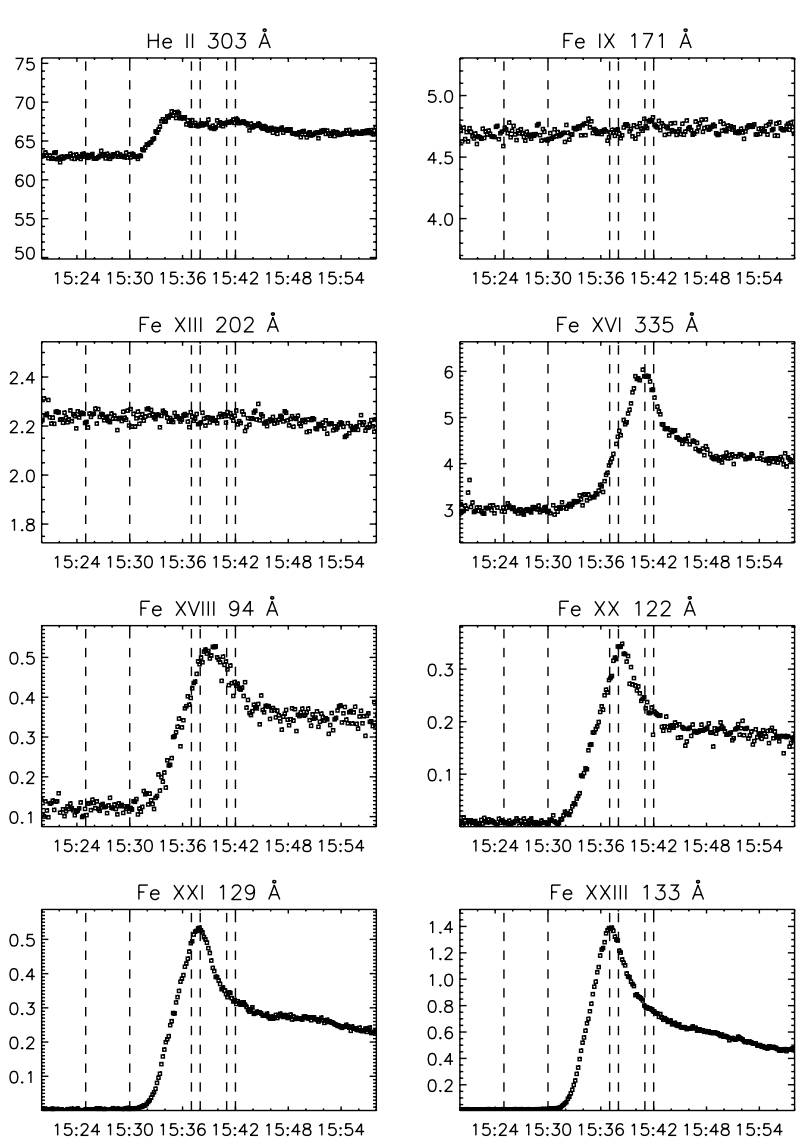

Fig. A.3. Light curves of several EVE lines during the M5.4 class flare of the 6th Nov. 2010. Irradiances are in $10^{8}$ phot $\mathrm{cm}^{-2} \mathrm{~s}^{-1}$, time in UT. The dashed lines indicate the three regions selected (pre-flare, gradual phase peak and gradual phase decline).
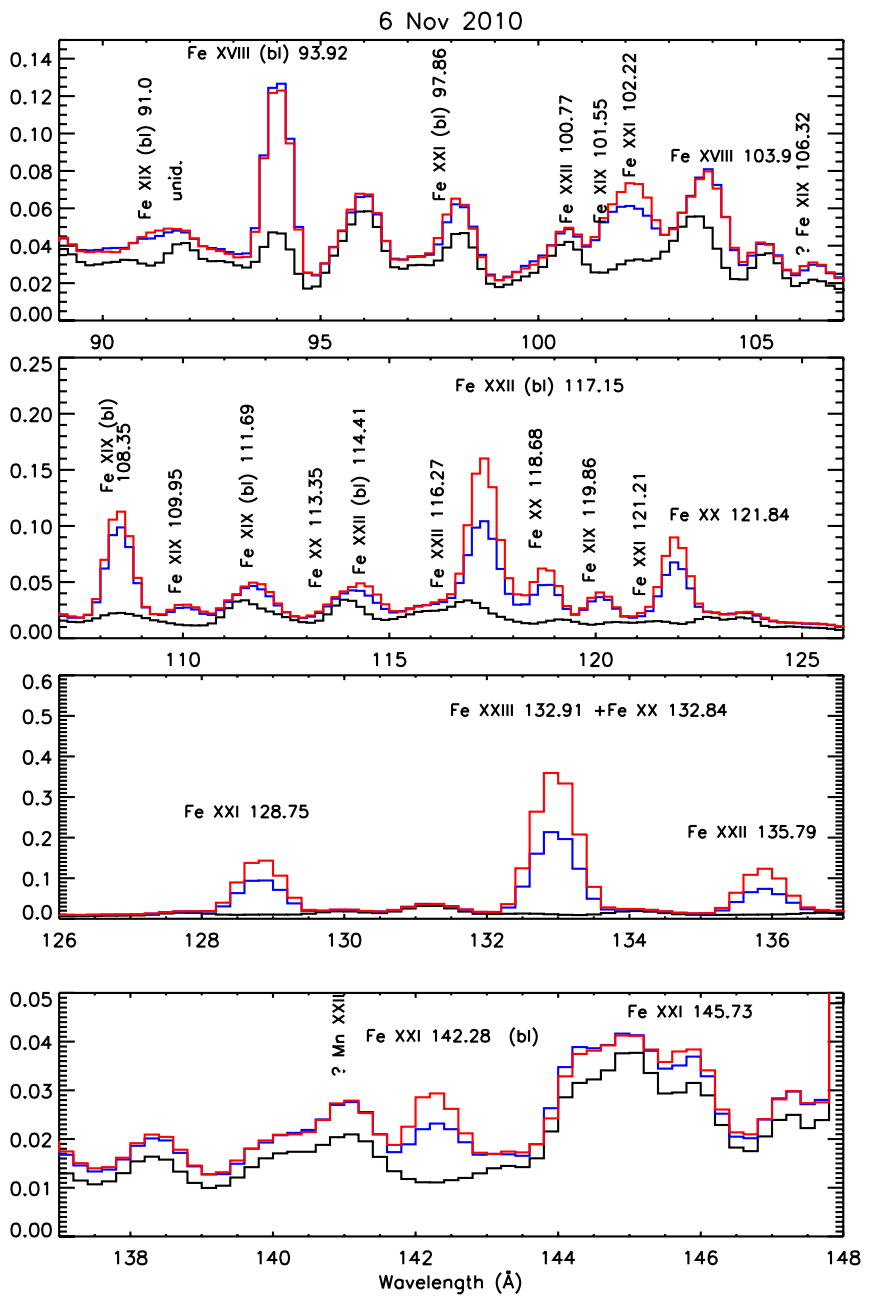

Fig. A.4. EVE spectra during the pre-flare (black), gradual phase peak (red), and gradual phase decline (blue) of the M5.4 class flare on 6 Nov. 2010 


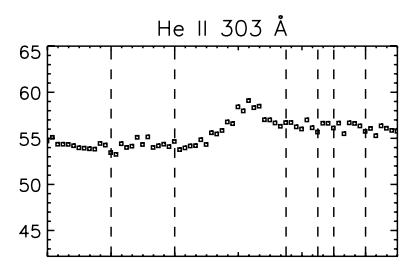

00:50 00:52 00:54 00:56 00:58 01:00
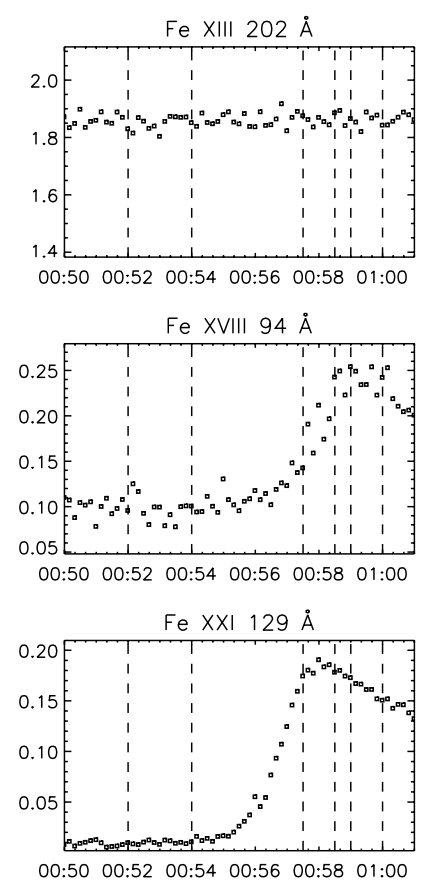

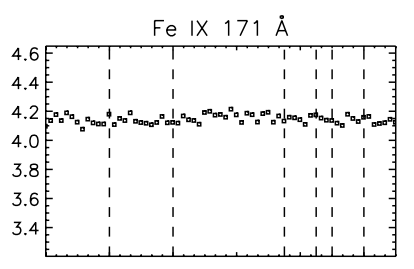

00:50 00:52 00:54 00:56 00:58 01:00
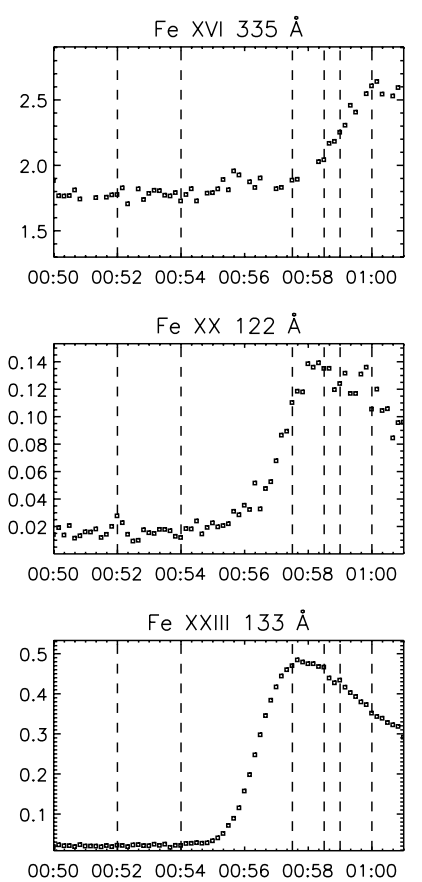

Fig. A.5. Light curves of several EVE lines during the M2.0 class flare on 12 June 2010. Irradiances are in $10^{8}$ phot $\mathrm{cm}^{-2} \mathrm{~s}^{-1}$, time in UT. The dashed lines indicate the three regions selected (pre-flare, gradual phase peak, and gradual phase decline).
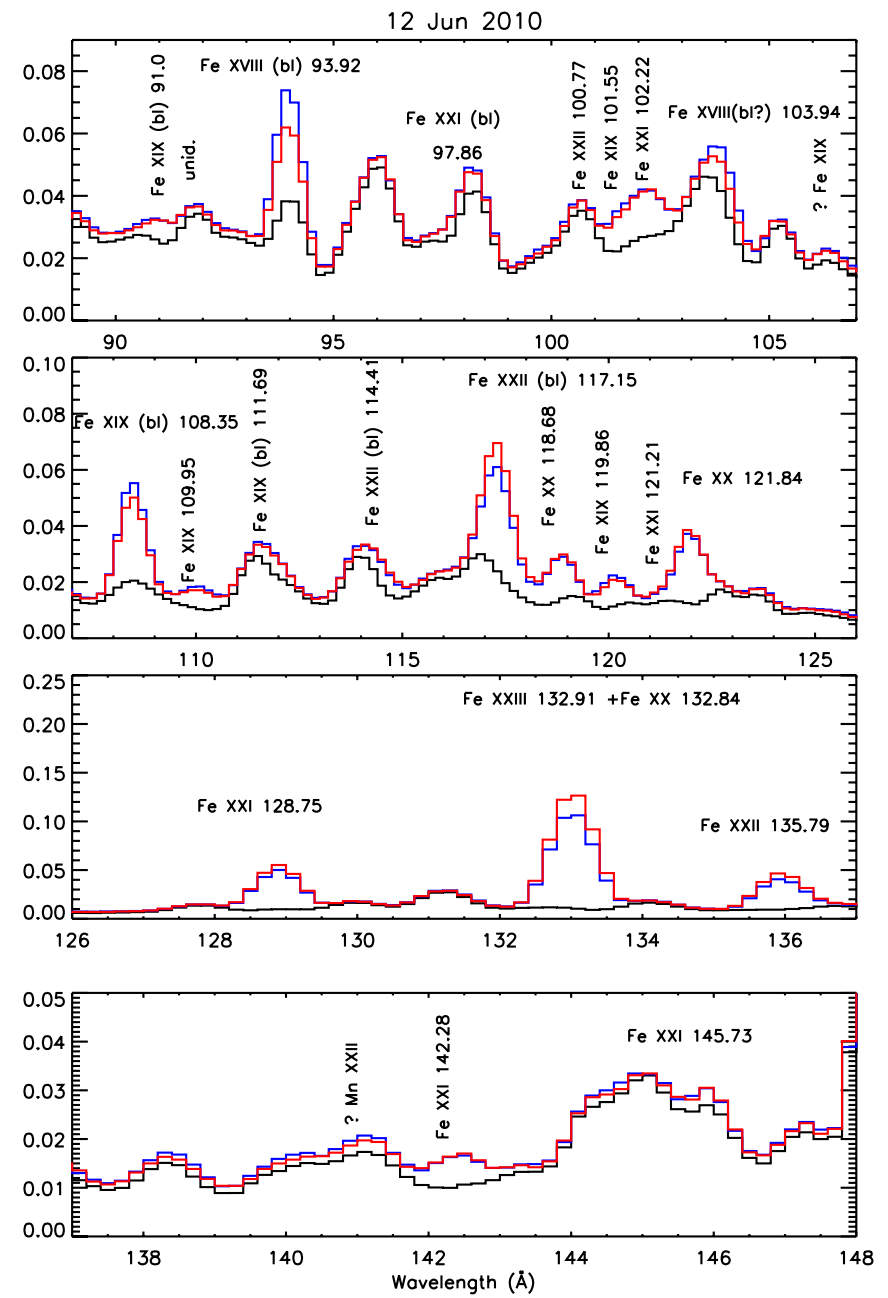

Fig. A.6. EVE spectra during the pre-flare (black), gradual phase peak (red), and gradual phase decline (blue) of the M2.0 class flare on 12 June 2010. 